\title{
Safety and efficacy of skin patches containing loxoprofen sodium in diabetic patients with overt nephropathy
}

\author{
Hisazumi Araki - Shogo Kuwagata $\cdot$ Mariko Soumura $\cdot$ Kosuke Yamahara \\ Yoshikata Morita - Shinji Kume · Keiji Isshiki • Shin-ichi Araki • \\ Atsunori Kashiwagi · Hiroshi Maegawa · Takashi Uzu
}

Received: 12 March 2013/Accepted: 29 July 2013/Published online: 7 August 2013

(C) The Author(s) 2013. This article is published with open access at Springerlink.com

\begin{abstract}
Background Because oral nonsteroidal anti-inflammatory drugs (NSAIDs) have adverse effects on kidney function, patients with kidney diseases are administered these drugs as transdermal patches. Little is known about the effects of NSAID patches on renal function. We therefore assessed the effects of topical loxoprofen sodium on kidney function in type 2 diabetic patients with overt nephropathy.

Methods Twenty patients with type 2 diabetes and overt proteinuria and with knee and/or low back pain were treated with skin patches containing $100 \mathrm{mg}$ loxoprofen on the knee or back for $24 \mathrm{~h}$ per day for 5 consecutive days. The degree of pain was assessed using a visual analogue scale (VAS). Blood and 24-h urine samples were obtained at baseline and at the end of the study. Glomerular filtration rate (GFR) was estimated from serum creatinine and cystatin $\mathrm{C}$ concentrations.

Results The 20 patients consisted of 11 males and 9 females, of mean age $61.6 \pm 13.9$ years. Loxoprofencontaining patches significantly reduced VAS pain without affecting blood pressure, GFR or urinary prostaglandin $\mathrm{E}_{2}$ concentration. Serum concentrations of loxoprofen and its active trans-OH metabolite did not correlate with GFR.

Conclusions Loxoprofen-containing patches do not affect renal function in type 2 diabetic patients with overt nephropathy over a short-term period. Long-term studies
\end{abstract}

Clinical Trial Registration: University Hospital Medical Information Network (No. UMIN000009520).

H. Araki $(\varangle) \cdot$ S. Kuwagata · M. Soumura · K. Yamahara ·

Y. Morita - S. Kume - K. Isshiki - S. Araki - A. Kashiwagi ·

H. Maegawa $\cdot$ T. Uzu

Department of Medicine, Shiga University of Medical Science,

Otsu, Shiga 520-2192, Japan

e-mail: haraki@belle.shiga-med.ac.jp are needed to clarify the safety of loxoprofen-containing patches in patients with chronic kidney diseases.

Keywords Skin patches - Loxoprofen - Diabetic nephropathy - Type 2 diabetes · Prostaglandin $\mathrm{E}_{2}$. GFR · NSAIDs

\section{Introduction}

Nonsteroidal anti-inflammatory drugs (NSAIDs) are widely used, with acknowledged efficacy and safety over a wide range of clinical conditions. Despite their many useful therapeutic applications, substantial evidence has shown that NSAIDs can have deleterious effects on kidney function. For example, a nested case-controlled study using the General Practice Research Database from the United Kingdom showed that NSAID users in the general population were at threefold greater risk for a first-ever diagnosis of clinical acute kidney injury (AKI) than nonNSAID users. In addition, history of heart failure, hypertension, and diabetes were associated with a greater risk of $\mathrm{AKI}$ in this population [1].

Combination therapy with NSAIDs and renin-angiotensin system (RAS) inhibitors increases the risk of kidney damage [2-4]. Since RAS inhibitors are recommended as first-line antihypertensive agents in patients with diabetes, patients with diabetic nephropathy who take NSAIDs tend to be at greater risk for NSAID-induced kidney damage.

NSAIDs can affect renal function by, for example, inhibiting the synthesis of important renal prostaglandins, especially those involved in solute homeostasis and maintenance of renal blood flow [5-8]. Prostaglandin $\mathrm{E}_{2}$ (PGE2) is the most abundant vasodilatory prostaglandin in the human renal vascular bed. NSAIDs decrease PGE2 
concentration by inhibiting cyclooxygenase-2 (COX-2). Adverse effects of NSAIDs may be avoided by administering these drugs as transdermal patches. These adhesive patches, which are applied to the skin at the site of pain, slowly release medication through the skin. Although NSAID patches are regarded as safe and are frequently used in patients with chronic kidney disease (CKD), the effects of NSAID patches on renal circulation in these patients have not been investigated. Loxoprofen-containing patches are one of the most widely used adhesive patches in Japan. We therefore analyzed the effects of topically applied loxoprofen sodium on kidney function in patients with diabetic nephropathy.

\section{Methods}

\section{Study design}

This open-label, single-arm, single-dose study was performed at the Shiga University of Medical Science Hospital. In patients with type 2 diabetes and overt proteinuria [urinary albumin-creatinine ratio (ACR) $>300 \mathrm{mg} / \mathrm{g}$ ] who had knee and/or low back pain were recruited. We enrolled 20 patients with type 2 diabetes complicated by diabetic nephropathy stage III to IV. Patients with diabetic nephropathy were classified by the Ministry of Health, Labour and Welfare of Japan [9]. The 20 patients consisted of 11 males and 9 females, ranging in age from 34 to 80 years [median age 61.6 years]. Patients previously treated with NSAIDs or with a history of hypersensitivity to NSAIDs were excluded. We also excluded those who underwent knee or spine surgery, and patients with hematologic disease, liver cirrhosis, heart failure or malignancy.

Adhesive skin patches containing $100 \mathrm{mg}$ loxoprofen (LX-P; Loxonin ${ }^{\circledR}$ tape) were applied to the back or knee of each patient, depending on the site of pain, for $24 \mathrm{~h}$ per day for five consecutive days (one patch per day). The degree of pain was assessed using a visual analogue scale (VAS) [10], consisting of a straight $10-\mathrm{cm}$ line, presenting a continuum of pain intensity, with 'no pain' at the bottom and 'pain as bad as it can be' at the top. Blood pressure was measured by an aneroid sphygmomanometer in the supine position before breakfast. The mean blood pressure values of 2 consecutive days before treatment were used as the baseline and the mean blood pressure value of days 4 and 5 were used as the end-point. Blood and urine samples were obtained under fasting conditions at baseline and at the end of the 5-day study period.

The estimated glomerular filtration rate (eGFRcre) of each patient was calculated using the simplified equation of the Japanese Society of Nephrology, a version of the Modification of Diet in Renal Disease study equation modified for Japanese patients [11]. GFR was also estimated from serum cystatin C concentrations (eGFRcys), as recently recommended by the Japanese Society of Nephrology [12]. HbA1c was measured using high-performance liquid chromatography and expressed as the National Glycohemoglobin Standardization Program (NGSP) equivalent value (\%), as recommended by the Japanese Diabetes Society. Serum concentrations of loxoprofen and its active, trans-OH metabolite were measured by liquid chromatography coupled with tandem mass spectrometry (LC/MS/MS) (Sumika Chemical Analysis Service, Ltd., Osaka, Japan). Urinary PGE2 concentrations were measured by a chemiluminescence immunoassay (SRL, Inc., Tokyo, Japan).

The study protocol was approved by the Ethics Committee of Shiga University of Medical Science (approval number: 22-83-1), and all participants provided written informed consent.

Statistical analysis

Data were analyzed using SPSS version 17.0 (SPSS, Tokyo, Japan). The distribution of variables was analyzed by checking histograms and normal plots of the data, and normality was tested using the Kolmogorov-Smirnov and Shapiro-Wilk tests. Student's $t$ test was used to compare values at different time points. Pearson's or Spearman's rank correlation coefficients were calculated to determine the correlations between variables. Values were expressed as mean $\pm \mathrm{SD}$, and $P<0.05$ was considered statistically significant.

\section{Results}

The 20 patients enrolled in this study consisted of 11 males and 9 females, ranging in age from 34 to 80 years (median age 61.6 years). The average height of the patients was $157.6 \pm 10.8 \mathrm{~cm}$, the average body weight was $69.8 \pm 18.6 \mathrm{~kg}$, and their average $\mathrm{HbA} 1 \mathrm{c}$ was $7.2 \pm$ $1.4 \%$. Their mean eGFRcre and eGFRcys were $24.8 \pm 17.7$ and $35.0 \pm 21.1 \mathrm{~mL} / \mathrm{min} / 1.73 \mathrm{~m}^{2}$, respectively. Two of the patients applied the LX-P on their knee and 18 applied the patch on their back.

Their mean systolic and diastolic blood pressure measurements at the end of the LX-P treatment were $133.7 \pm 21.5$ and $73.2 \pm 11.7 \mathrm{mmHg}$, respectively. Systolic and diastolic blood pressure at the end of treatment did not differ significantly from baseline $(P=0.211$ and $P=0.843$, respectively). Pain assessed on a 10-point VAS was significantly reduced by LX-Ps (Fig. 1a), whereas renal function, assessed by eGFRcre and eGFRcys, was not affected (Fig. 1b, c). In addition, urinary PGE2 
Fig. 1 Effects of topically administered LX-Ps on (a) pain VAS, (b) eGFRcre,

(c) eGFRcys, and (d) urinary PGE2. $* * P<0.01$
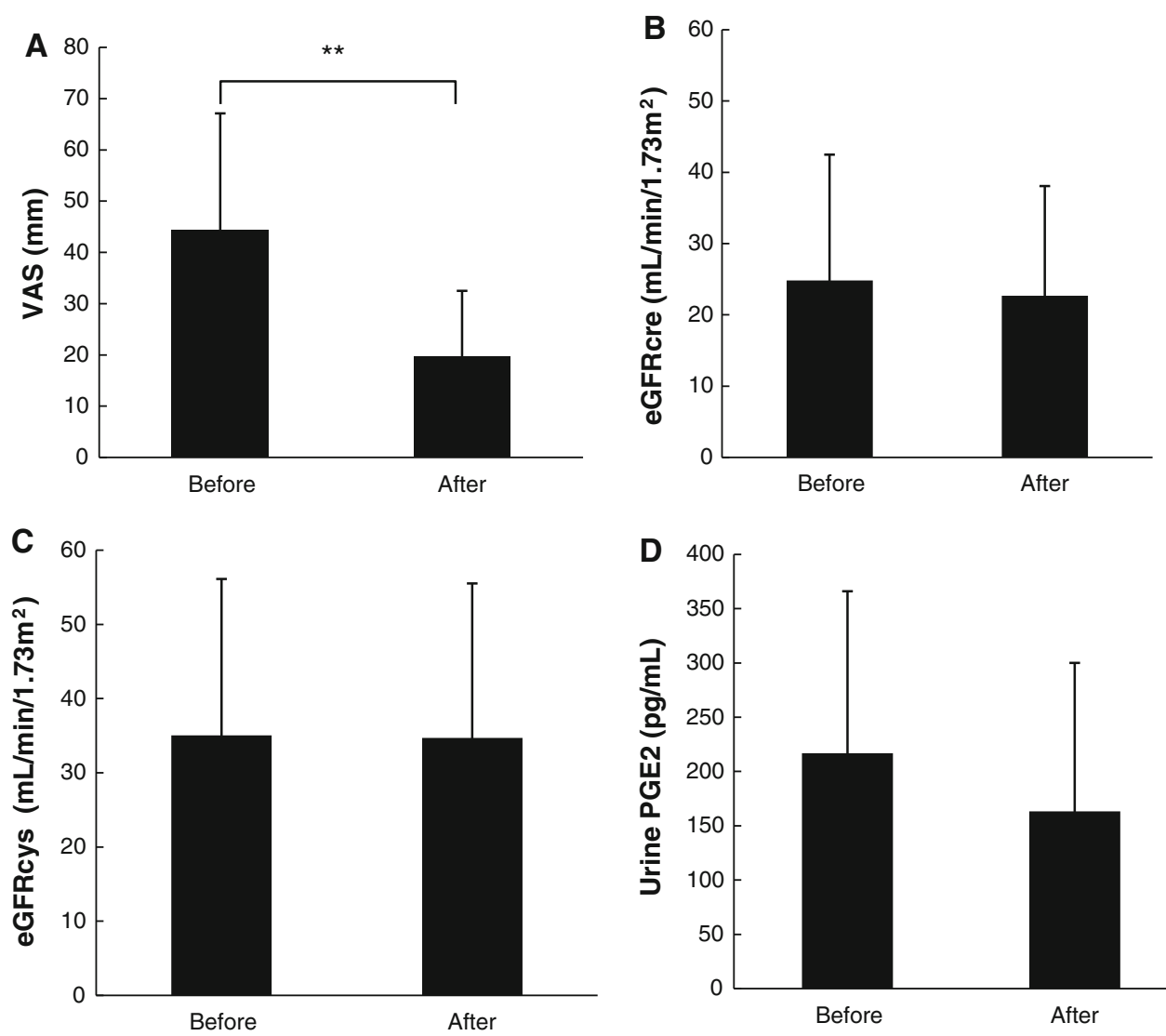

concentrations did not change from baseline to the end of therapy (Fig. 1d). These results indicated that, in patients with type 2 diabetes and overt proteinuria, LX-Ps reduced pain without affecting renal microcirculation.

The mean $\pm \mathrm{SD}$ serum concentrations of loxoprofen and its trans-OH metabolite at the end of the 5-day LX-P treatment period were $100.2 \pm 75.0$ and $50.4 \pm 45.2 \mathrm{ng} / \mathrm{mL}$, respectively. These concentrations did not correlate with renal function (Fig. 2a, b).

PGE2 concentrations in fasting urine before and after the administration of LX-Ps did not differ significantly $(216.9 \pm 149.3$ and $163.3 \pm 136.9 \mathrm{pg} / \mathrm{mL}, \quad P=0.23)$ (Fig. 1d). Moreover, there was no correlation between the concentration of PGE2 and eGFRcys, either before $(r=-0.16, P=0.51)$ or after $(r=-0.14, P=0.55)$ treatment with LX-Ps (data not shown).

\section{Discussion}

Although the serum concentrations of loxoprofen sodium have been measured following oral administration in patients without renal impairment, these concentrations were not measured in patients with renal impairment. To our knowledge, this study is the first to evaluate serum concentrations of loxoprofen sodium and urinary concentrations of PGE2 following the administration of LX-Ps to patients with diabetic nephropathy.

We found that short-term administration of LX-Ps was effective in treating knee and lower back pain in Japanese patients with diabetic nephropathy, without negatively affecting renal function. All 20 of our patients had overt protein in urine, but their eGFRcre ranged from normal $\left(>60 \mathrm{~mL} / \mathrm{min} / 1.73 \mathrm{~m}^{2}\right)$ to severe renal impairment $\left(<30 \mathrm{~mL} / \mathrm{min} / 1.73 \mathrm{~m}^{2}\right)$. Serum concentration of loxoprofen sodium and its trans-OH metabolite following a single oral dose of $60 \mathrm{mg}$ have been reported to be $5.04 \pm 0.27$ and $0.85 \pm 0.02 \mu \mathrm{g} / \mathrm{mL}$, respectively [13]. We found that both serum concentrations were much lower, 100.2 \pm 75.0 and $50.4 \pm 45.2 \mathrm{ng} / \mathrm{mL}$, respectively, after the application of transdermal LX-Ps. Moreover, these patches had no effect on PGE2 concentrations. Taken together, these results suggest that topically administered loxoprofen sodium was safer for patients with renal impairment than the orally administered agent.

Loxoprofen sodium and its trans-OH metabolite are both metabolized in and secreted by the liver and kidneys, suggesting that, in patients with renal impairment, their serum concentrations would be higher in patients with AKI than in those with normal renal function. To assess whether serum concentrations of these molecules differed according to renal function, we examined the relationship of each to 
Fig. 2 Correlations between eGFRcys and the absorption of loxoprofen sodium. The correlation of eGFRcys and serum concentration of (a) loxoprofen sodium ( $r=0.15, P=0.53)$ and (b) the trans-OH metabolite of loxoprofen sodium $(r=-0.073, P=0.76)$
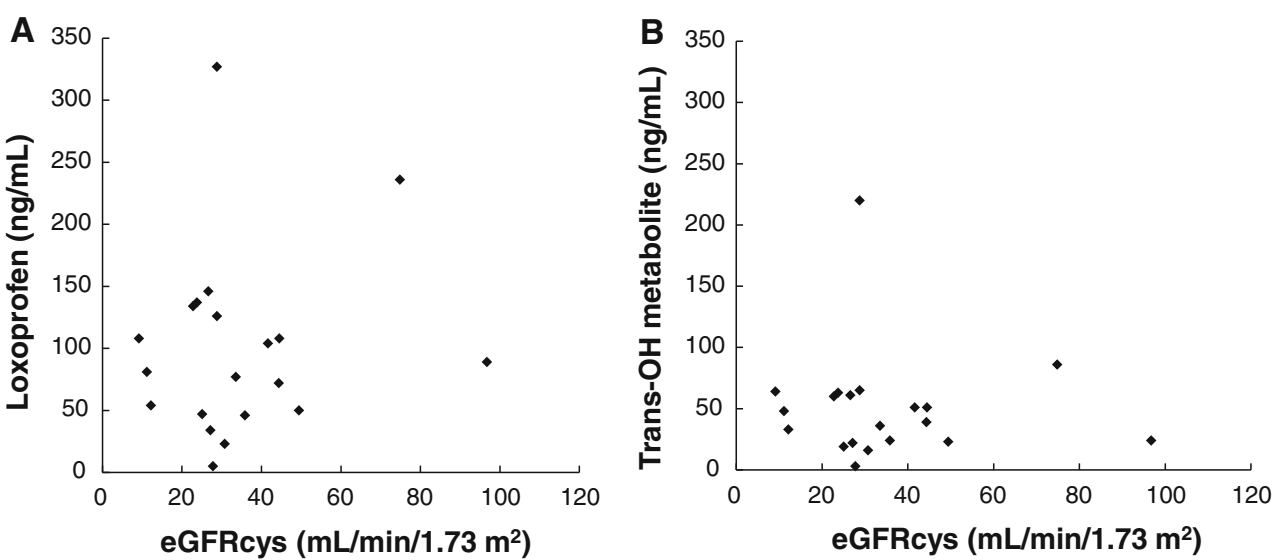

eGFRcys. However, we did not detect any correlations. These findings indicated that loxoprofen sodium and its active metabolite were not increased in patients with severe renal impairment. This suggests that the absorption of loxoprofen sodium by the systemic circulation is lower when this agent is administered topically than orally, and is therefore not altered by renal function. We predict that the concentration of loxoprofen sodium and its trans-OH metabolite are in equilibrium after five consecutive days, but the details of their pharmacokinetics in patients with renal impairment is still unknown.

We analyzed the correlation between the concentration of loxoprofen sodium or its trans-OH metabolite and urinary PGE2. There was no correlation between the concentrations of loxoprofen sodium and urinary PGE2 $(P=0.345)$, or between the trans-OH metabolite and urinary PGE2 $(P=0.370)$ (data not shown). We postulated that this is because the concentrations of loxoprofen sodium and its trans-OH metabolite were so low and in such a narrow range.

NSAIDs are associated with elevated blood pressure and a higher incidence of hypertension [14-19] because they inhibit the production of prostaglandins. However, we found that topically administered loxoprofen sodium did not significantly affect systolic or diastolic blood pressure, likely because it does not decrease prostaglandins.

In conclusion, in contrast to orally administered loxoprofen sodium, topically administered LX-Ps did not increase serum loxoprofen concentrations or decrease urinary PGE2 concentrations in Japanese patients with type 2 diabetes and renal impairment. Topical LX-Ps had no effect on renal function or on blood pressure in these patients. Although our study was limited by the small number of patients, topical LX-Ps showed good short-term safety and efficacy results in patients with diabetic nephropathy. However, additional large-scale and longterm studies are needed to clarify the safety of LX-Ps in patients with chronic kidney diseases. Furthermore, the effect of LX-Ps in patients on dialysis therapy is currently unclear, suggesting the need for further studies to clarify these effects.

Acknowledgments The authors acknowledge the assistance of Ayano Takagi, Shinya Ono and Syohei Yoshida at Shiga University of Medical Science.

Conflict of interest The authors declare no conflict of interest.

Open Access This article is distributed under the terms of the Creative Commons Attribution License which permits any use, distribution, and reproduction in any medium, provided the original author(s) and the source are credited.

\section{References}

1. Huerta C, Castellsague J, Varas-Lorenzo C, Garcia Rodriguez LA. Nonsteroidal anti-inflammatory drugs and risk of ARF in the general population. Am J Kidney Dis: Off J Natl Kidney Found. 2005;45(3):531-9.

2. Nitsch D, Tomlinson LA. Safety of co-prescribing NSAIDs with multiple antihypertensive agents: triple drug combinations are associated with increased hospital admission for acute kidney injury, but questions remain. BMJ. 2013;346:e8713.

3. Loboz KK, Shenfield GM. Drug combinations and impaired renal function - the 'triple whammy'. Br J Clin Pharmacol. 2005;59(2): 239-43.

4. Fournier JP, Sommet A, Durrieu G, Poutrain JC, Lapeyre-Mestre M, Montastruc JL. Drug interactions between antihypertensive drugs and non-steroidal anti-inflammatory agents: a descriptive study using the French Pharmacovigilance database. Fund Clin Pharmacol 2012. DOI: $10.1111 /$ fcp. 12014

5. Clive DM, Stoff JS. Renal syndromes associated with nonsteroidal antiinflammatory drugs. New Engl J Med. 1984;310(9): 563-72.

6. Garella S, Matarese RA. Renal effects of prostaglandins and clinical adverse effects of nonsteroidal anti-inflammatory agents. Medicine. 1984;63(3):165-81.

7. Carmichael J, Shankel SW. Effects of nonsteroidal anti-inflammatory drugs on prostaglandins and renal function. Am J Med. 1985;78(6 Pt 1):992-1000.

8. Patrono C, Dunn MJ. The clinical significance of inhibition of renal prostaglandin synthesis. Kidney Int. 1987;32(1):1-12.

9. Stage classification of diabetic nephropathy: report of the Ministry of Health and Welfare, Japan (in Japanese); 1991 pp. 251-256. 
10. Scott J, Huskisson EC. Graphic representation of pain. Pain. 1976;2(2):175-84.

11. Matsuo S, Imai E, Horio M, Yasuda Y, Tomita K, Nitta K, et al. Revised equations for estimated GFR from serum creatinine in Japan. Am J Kidney Dis: Off J Natl Kidney Found. 2009;53(6):982-92.

12. Japanese Society of Nephrology ed. Clinical Practice Guidebook for Diagnosis and Treatment of Chronic Kidney Disease 2012. Tokyo: Tokyo igaku sya; 2012.

13. Naganuma HMY, Kawahara Y. Study of pharmacokinetics following oral administration of loxoprofen sodium (CS-600) in humans. Rinsho Iyaku. 1986;2(9):1219-37.

14. Aljadhey H, Tu W, Hansen RA, Blalock SJ, Brater DC, Murray MD. Comparative effects of non-steroidal anti-inflammatory drugs (NSAIDs) on blood pressure in patients with hypertension. BMC Cardiovasc Disord. 2012;12:93.

15. Johnson AG, Simons LA, Simons J, Friedlander Y, McCallum J. Non-steroidal anti-inflammatory drugs and hypertension in the elderly: a community-based cross-sectional study. Br J Clin Pharmacol. 1993;35(5):455-9.

16. Dedier J, Stampfer MJ, Hankinson SE, Willett WC, Speizer FE, Curhan GC. Non-narcotic analgesic use and the risk of hypertension in US women. Hypertension. 2002;40(5):604-8 discussion $1-3$.

17. Kurth T, Hennekens CH, Sturmer T, Sesso HD, Glynn RJ, Buring $\mathrm{JE}$, et al. Analgesic use and risk of subsequent hypertension in apparently healthy men. Arch Intern Med. 2005;165(16):1903-9.

18. Solomon DH, Schneeweiss S, Levin R, Avorn J. Relationship between COX-2 specific inhibitors and hypertension. Hypertension. 2004;44(2):140-5.

19. Wang J, Mullins CD, Mamdani M, Rublee DA, Shaya FT. New diagnosis of hypertension among celecoxib and nonselective NSAID users: a population-based cohort study. Ann Pharmacother. 2007;41(6):937-43. 